\title{
Improved Model for Beam-Wave Interaction with Ohmic Losses and Reflections of Sheet Beam Traveling Wave Tubes
}

\author{
Hanwen Tian, Ningjie Shi, Zhanliang Wang, Shaomeng Wang, Member, IEEE, Zhaoyun \\ Duan, Senior Member, IEEE, Huarong Gong, Zhigang Lu, Claudio Paoloni, Senior \\ Member, IEEE, Jinjun Feng, Senior Member, IEEE, and Yubin Gong, Member, IEEE
}

\begin{abstract}
In this paper, an improved model for the beam-wave interaction of sheet beam in traveling wave tubes (TWTs) considering ohmic losses and reflections is presented. The ohmic losses are obtained by field analysis and equivalent method. The space charge magnetic field is derived from the active Helmholtz's equation. An algorithm to obtain the S-matrix by the equivalent circuit method is presented. The relativistic Boris method is applied to accelerate macroparticles. The exchanged power is computed by the work the electromagnetic field applied to the macroparticles. The theoretical model is applied for validation to a G-band staggered double vane TWT and validated by comparison with CST Particle Studio and simulations without losses and reflections. The convergence of this algorithm is also discussed. The simulation time of the model is substantial faster than 3D Particle-in-cell (PIC) simulations.
\end{abstract}

Index Terms-beam-wave interaction, losses, reflections, sheet beam TWT

\section{INTRODUCTION}

$\mathrm{O}$ HMIC losses and reflections of slow wave structures (SWSs) are important parameters for a reliable and accurate simulation of TWTs. This is particularly important at millimeter wave $(30-300 \mathrm{GHz})$ and Terahertz $(\mathrm{THz}, 300$ $\mathrm{GHz}-1000 \mathrm{GHz}$ ) frequencies due to the small dimensions that affects the fabrication accuracy and the decreasing skin depth that requires high quality surface roughness to keep losses low.

The 3D fast time-domain nonlinear algorithm (FTDNA-3D) presented in [1] is a PIC-method-based approach for

This work was supported in part by the China Scholarship Council, in part by the National Natural Science Foundation of China under Grant No. 61921002 and No. 61988102.

H. Tian (e-mail: hrtianhanwen@163.com), N. Shi (e-mail: elizabethshi0320@163.com), Z. Wang (e-mail: wangzl@uestc.edu.cn), S. Wang (e-mail: wangsm@uestc.edu.cn), Z. Duan (e-mail: zhyduan@uestc.edu.cn), H. Gong (e-mail: hrgong@uestc.edu.cn), Z. Lu (e-mail: Izhgchnn@uestc.edu.cn), and Y. Gong (e-mail: ybgong@uestc.edu.cn) are with National Key Laboratory of Science and Technology on Vacuum Electronics, School of Electronic Science and Engineering, University of Electronic Science and Technology of China, Chengdu 610054, China.

H. Tian, and C. Paoloni (e-mail: c.paoloni@lancaster.ac.uk) are with Engineering Department, Lancaster University, Lancaster, UK.

J. Feng (e-mail: fengjinjun@tsinghua.org.cn) is with Beijing Vacuum Electronics Research Institute, Beijing 100015, China

Corresponding author: Yubin Gong. beam-wave interaction in the sheet beam SWSs. Using the specific electromagnetic properties in the sheet beam SWSs, this algorithm significantly simplifies the simulation compared with the traditional 3D electromagnetic simulation method based on finite difference time domain (FDTD) method. It substantially reduces the simulation time but limited simulation accuracy.

The FTDNA-3D algorithm needs further improvement to provide accurate and useful results as it is under the hypothesis of perfect electric conductor (PEC). However, losses affect the TWT performances and should be included in the model. To introduce the losses, the magnetic field in the SWS must be derived. Secondly, the FTDNA-3D does not model well the reflections of the SWS, another important parameter for the accurate modeling of the TWT performances. A theory for introducing the reflections in FTDNA-3D has to be found.

This paper proposes a time domain self-consistence solution to improve FTDNA-3D algorithm for simulating the beam-wave interaction including the effect of ohmic losses and reflections of SWSs. Compared with [1], the two models share almost identical method for electric field computation, while the new model considers the losses and reflections. The models of magnetic field, losses and reflections are presented in this paper originally. As a verification example, a staggered double vane waveguide is considered [2-4]. Methods for calculation of the electromagnetic fields, particle behavior and energy exchange are also presented.

In Section II and III, the method for the derivation of the losses and reflections will be presented. In Section IV, the equations of particle movement and energy exchange are presented. In section $\mathrm{V}$, the flow chart of this algorithm is discussed. In Section VI, the comparison of the simulations of a G-band staggered double vane TWT by CST particle studio, the proposed method and simulation without losses and reflections is presented. The convergences, simulation time and some other discussions are also presented. The conclusions are drawn in Section VII.

\section{LOSSES}

The metal walls of a SWS cannot be considered made of perfect conductor due to the not perfect surface finishing that reduces the conductivity. This increases the ohmic losses that affects the electrical behavior of the SWS. The metal losses occur both inside and outside the beam channel. 
The metal losses inside the beam channel are computed by the magnetic field directly. The magnetic field in the beam channel has to be computed as the superposition of circuit and space charge magnetic field, established by the surface current on the conductor walls and the electron beam in the beam channel, respectively. The metal losses outside the beam channel could be related with the power propagating in the SWS by equivalent circuit method.

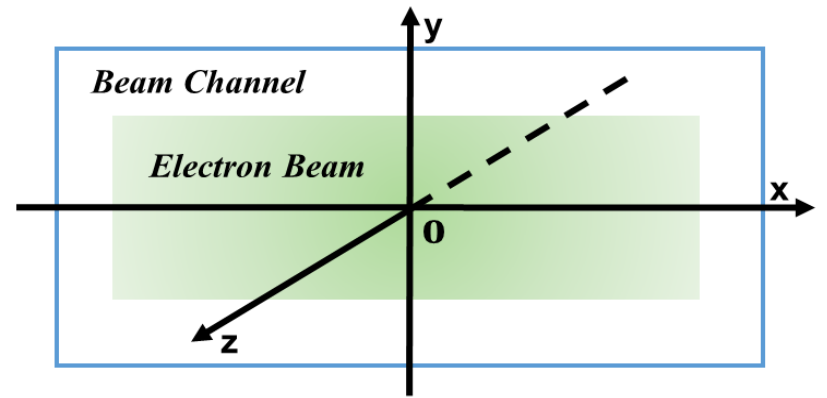

Fig. 1. Schematic of beam channel, sheet electron beam in the emitting surface, and coordinate system.

\section{A. Inside the Beam Channel}

The beam channel and the coordinate system are shown in Fig.1. The x-component of circuit electric field is assumed as zero [5-6]. The mode of wave is assumed as $\mathrm{TE}_{10}$ mode.

At first, we consider the circuit field of the SWS. As we know, the Pierce impedance of $n^{\text {th }}$ harmonic is [7]

$$
k_{c n}(x, y)=\frac{\left|E_{c z n}(x, y)\right|^{2}}{2 \beta_{n}^{2} P}
$$

where $E_{c z n}$ is the amplitude of z-component of $n^{\text {th }}$ harmonic of circuit electric field, $\beta_{n}$ is the phase constant of $n^{\text {th }}$ harmonic and $P$ is the power.

In case of losses, the z-component of circuit electric field is

$$
E_{c z}(x, y, z)=\sum_{n} \sqrt{2 k_{c n}(x, y) \beta_{n}^{2} P} e^{-\gamma_{n} z}
$$

where $\gamma_{n}$ is the complex propagation constant of $n^{\text {th }}$ spatial harmonic. The factor $e^{j \omega t}$ is omitted for simplicity despite the field is assumed as time harmonic.

The relation between $\mathrm{x}$-component of circuit magnetic field and z-component of circuit electric field is [5]

$$
E_{c z}=-\frac{j \omega \mu}{h^{2}} \frac{\partial H_{c x}}{\partial y}
$$

where $\omega$ is the angular frequency, $\mu$ is the permeability, and $h$ is a constant related to the mode of the propagating wave.

$$
h^{2}=\left(\frac{\omega}{c}\right)^{2}-\left(\frac{\pi}{w}\right)^{2}
$$

where $c$ is the light velocity in free space, and $w$ is the width of sheet beam channel.

From (2) and (3), we obtain

$$
H_{c x}=\frac{j h^{2}}{\omega \mu} \int E_{c z} d y=\sum_{n} \frac{j h^{2}}{\omega \mu} \beta_{n} \sqrt{2 P} e^{-\gamma_{n} z} \int \sqrt{k_{c n}} d y
$$

According to the relations between the $\boldsymbol{H}_{c x}$ and components of the electromagnetic wave shown in the (A1) - (A3) in appendix, we have

$$
\begin{gathered}
H_{c y}=\frac{j}{\omega \mu} \frac{\partial E_{c z}}{\partial x}=\sum_{n} \frac{j}{\omega \mu} \beta_{n} \sqrt{2 P} \frac{\partial \sqrt{k_{c n}}}{\partial x} e^{-\gamma_{n} z} \\
H_{c z}=\frac{1}{j \omega \mu} \frac{\partial E_{c y}}{\partial x}=\sum_{n} \frac{\beta_{n} \gamma_{n}}{j \omega \mu} \sqrt{2 P} e^{-\gamma_{n} z} \int \frac{\partial \sqrt{k_{c n}}}{\partial x} d y \\
E_{c y}(x, y, z)=\sum_{n} \gamma_{n} \int \sqrt{2 k_{c n}(\mathrm{x}, \mathrm{y}) \mathrm{P}} d y e^{-\gamma_{n} z}
\end{gathered}
$$

Secondly, we consider the space charge field in SWS.

As shown in appendix, substituting (A6) to (A4), we obtain

$$
\begin{gathered}
E_{s z}=\sum_{n} \frac{1}{\gamma_{e n}^{2}+k^{2}}\left(\mu \frac{\partial J_{z n}}{\partial t}+\frac{\partial}{\partial z} \frac{\rho_{n}}{\varepsilon}\right) \\
H_{s z}=\sum_{n} \frac{1}{\gamma_{e n}^{2}+k^{2}}\left(\frac{\partial J_{x n}}{\partial y}-\frac{\partial J_{y n}}{\partial x}\right)
\end{gathered}
$$

Solving the Maxwell equations under time harmonic conditions shown in appendix (A9), we obtain the transversal components of space charge electric and magnetic field.

$$
\begin{aligned}
& H_{s x}=\sum_{n} \frac{1}{k^{2}+\gamma_{e n}^{2}}\left[\mathrm{j} \omega \varepsilon \frac{\partial E_{s z n}}{\partial y}-\gamma_{e n}\left(\mathbf{J}_{y n}+\frac{\partial H_{s z n}}{\partial x}\right)\right] \\
& H_{s y}=\sum_{n} \frac{1}{k^{2}+\gamma_{e n}^{2}}\left[\gamma_{e n}\left(\mathbf{J}_{x n}-\frac{\partial H_{s z n}}{\partial y}\right)-\mathrm{j} \omega \varepsilon \frac{\partial E_{s z n}}{\partial x}\right] \\
& E_{s x}=\sum_{n} \frac{1}{k^{2}+\gamma_{e n}^{2}}\left[j \omega \mu\left(\mathbf{J}_{x n}-\frac{\partial H_{s z n}}{\partial y}\right)-\gamma_{e n} \frac{\partial E_{s z n}}{\partial x}\right] \\
& E_{s y}=\sum_{n} \frac{1}{k^{2}+\gamma_{e n}^{2}}\left[j \omega \mu\left(\mathbf{J}_{y n}+\frac{\partial H_{s z n}}{\partial x}\right)-\gamma_{e n} \frac{\partial E_{s z n}}{\partial y}\right]
\end{aligned}
$$

Superposing the circuit and space charge magnetic field, we obtain the magnetic field in the beam channel. Then, we have the expression of the metal losses for unit length as:

$$
P_{l 1}=\frac{R_{s}}{2} \int_{S}\left|H_{t}\right|^{2} d s
$$

where $H_{t}$ is the tangential magnetic field, and $R_{s}$ is the surface resistance inside the beam channel. Under smooth surface assumption, we have

$$
R_{s}=\sqrt{\frac{\omega \mu}{2 \sigma}}
$$

Where $\sigma$ is the conductivity of the beam channel.

\section{B. Outside the Beam Channel}

The metal losses outside the beam channel always depend on the shape and dimensions of the SWS. In the following, we will consider a staggered double vane SWS for method validation. Its basic unit cell could be regarded as a T-type junction of two rectangular waveguides, and one of them is cut off by a metal plane, as seen in Fig.2(a). For the lossy case, the metal plane is modeled with an impedance. The equivalent circuit of the lossy case of one basic unit cell of staggered double vane SWS results as in Fig.2(b) [8].

The impedance $Z_{L}$ in Fig.2(b) is due to the metal losses outside the beam channel. Its value is the surface impedance of the metal. The value of $B_{a}-B_{d}$ could be found in appendix (A10).

Analyzing the circuit in Fig.2(b), the relation between the power losses in $Z_{L}\left(P_{L}\right)$ and propagated in beam channel $\left(P_{i}\right)$ is 


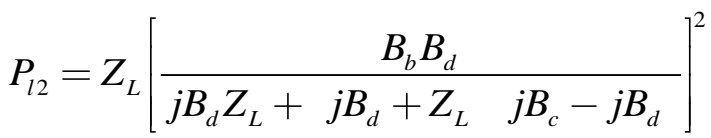

$$
\begin{aligned}
& \times \frac{B_{a}^{2} Y_{0}^{2}+2 j B_{a} Y_{0}-1}{Y_{0} B_{a}^{2}} P_{i}
\end{aligned}
$$

where $Y_{0}$ is the port admittance, and

$$
Y_{0}=\frac{2 w}{\pi b} \frac{\beta}{k \eta}
$$

where $b$ is the height of beam channel, and $\eta$ is the intrinsic impedance in vacuum.

\section{RefLECTIONS}

Reflections cannot be avoided in a SWS due to inaccuracy of fabrication, discontinuities and not perfect matching of the couplers. They could significantly affect the output power.

We can calculate the S-matrix of the staggered double vane SWS by the equivalent circuit method as shown in Fig.3. The SWS is regarded as a cascaded networks of transmission lines (for the continuous part) and admittances (for the discontinuous part). The boundaries of both ends are assumed as periodic boundary. The value of $B_{B}$ and $B_{D}$ in Fig.3 can be obtained as in [8].

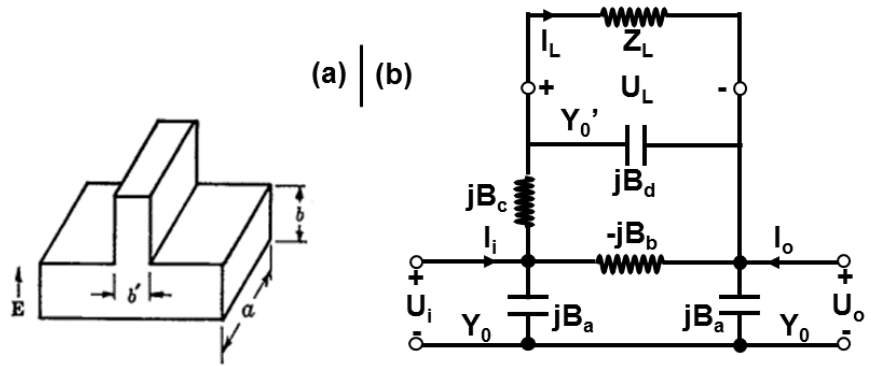

Fig.2. (a) Schematic of T-type junction of two rectangular waveguides; (b) the equivalent circuit of staggered double vane SWS [8].

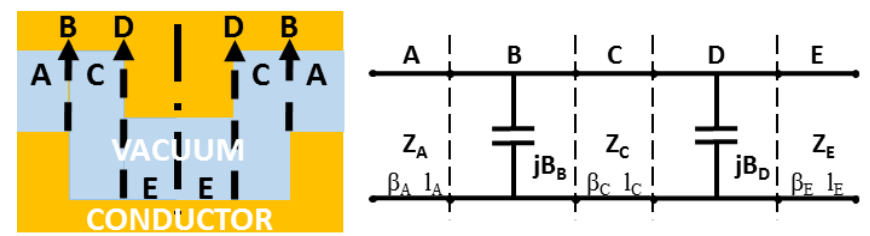

Fig. 3. Schematic of equivalent circuit model (half period) of staggered double vane SWS.

The transmission matrices of transmission line and admittances are shown as follow.

$$
\begin{gathered}
T_{t}=\left(\begin{array}{cc}
\cos \beta l & j Z_{0} \sin \beta l \\
j Y_{0} \sin \beta l & \cos \beta l
\end{array}\right) \\
T_{a}=\left(\begin{array}{cc}
1 & 0 \\
j B & 1
\end{array}\right)
\end{gathered}
$$

The transmission matrix of one period of staggered double vane SWS is

$$
[\mathrm{T}]=[\mathrm{A}][\mathrm{B}][\mathrm{C}][\mathrm{D}][\mathrm{E}][\mathrm{E}][\mathrm{D}][\mathrm{C}][\mathrm{B}][\mathrm{A}]
$$

The S-matrix of single period could be obtained from the transmission matrix in (19). In order to obtain the S-matrix of several periods, the relationship between the S-matrices elements of network $(\boldsymbol{S})$ and its subnetwork $(\boldsymbol{U})$ is given as follow.

$$
\begin{gathered}
s_{11}=\frac{u_{11}(1-|U|)}{1-u_{11} u_{22}} \\
s_{12}=s_{21}=\frac{u_{12}^{2}}{1-u_{11} u_{22}} \\
s_{22}=\frac{u_{22}(1-|U|)}{1-u_{11} u_{22}}
\end{gathered}
$$

\section{Particle Movement And Energy Exchange}

The accurate modelling of the particle movement is a fundamental component of PIC methods. The electrons are modelled by macroparticles to keep the simulation domain limited. The electromagnetic field derived in the previous section is used to accelerate the macroparticles. Based on the movement of macroparticles, the energy exchanged between particles and electric field could be obtained. In this section, the Boris method [9-11] considering relativistic effect [12] is used to describe the particle movement, and the property of conservative field is used to calculate the energy exchanged between particles and field.

In order to avoid the error caused by relativistic effect, and include high-voltage cases, we define that

$$
\vec{u}_{k \pm \frac{1}{2}}=\vec{v}_{k \pm \frac{1}{2}} \gamma_{k \pm \frac{1}{2}}
$$

where $\boldsymbol{u}$ is normalized velocity of particles, $\boldsymbol{v}$ is real velocity of particles, $\gamma$ is the Lorentz factor and $k$ is the time step number.

The relativistic Lorentz's difference equation is

$$
\frac{\vec{u}_{k+1 / 2}-\vec{u}_{k-1 / 2}}{\Delta t}=\eta\left(\overrightarrow{\mathrm{E}}_{k}+\frac{\vec{u}_{k+1 / 2}+\vec{u}_{k-1 / 2}}{2} \times \mu \vec{H}_{k}\right)
$$

where $\eta$ is the charge-to-mass ratio of electron, $\Delta t$ is the time step length, $\boldsymbol{E}_{\boldsymbol{k}}$ and $\boldsymbol{H}_{\boldsymbol{k}}$ are electric and magnetic field at time step $k$, respectively.

By the well-known relativistic Boris method, we can obtain the velocity of macroparticles at time step $k+1 / 2$ from the velocity of time step $k-1 / 2$. And the displacement of time step $k$ is

$$
\Delta \vec{s}_{k}=\Delta t \frac{\overrightarrow{\mathrm{v}}_{k+\frac{1}{2}}+\overrightarrow{\mathrm{v}}_{k-\frac{1}{2}}}{2}
$$

The work the electric field applied to the macroparticles will be converted to the energy of macroparticles. Thus, the exchanged power at time step $k$ is

$$
\Delta P_{k}=\frac{\Delta \varepsilon_{k}}{\Delta t}=\frac{\rho\left(\overrightarrow{\mathrm{E}}_{k} \cdot \Delta \vec{s}_{k}\right)}{\Delta t}
$$

This exchanged power is used to obtain the power in the SWS at time step $k+1$, which is used to compute the field at time step $k+1$.

\section{ALgorithm FLOW}

The input parameters are input power $P_{i}$, frequency of wave $f$, beam current $I$, beam voltage $U$, charge and current density of beam $\rho$ and $J$, conductivity of the conductor with limited 
conductivity $\sigma$, the preset simulation time $t$ and the dimensions of the SWS. The Pierce impedance $k_{c}$, phase constant $\beta_{n}$ could be calculated based on the dimensions of SWS.

The second step is the calculation of the electric and magnetic fields. With the value of power $P$, phase constant $\beta_{n}$, and Pierce impedance $k_{c}$, using (2) and (8), the circuit electric field will be obtained. The space charge electric field is obtained from the current density and charge density of beam $\rho$ and $J$, using (9), (13) and (14). Superposing the circuit and the space charge electric fields, we will get the electric field in the SWS at time step $k$, marked as $\boldsymbol{E}_{\boldsymbol{k}}$ *. Then, by using (5), (6) and (7), the circuit magnetic field is obtained. The space charge magnetic field is derived from (10), (11) and (12). Superposing the circuit and space charge magnetic field, we will get the magnetic field established in the SWS at time step $k$, marked as $\boldsymbol{H}_{\boldsymbol{k}}$ *

Then, with the data of electric and magnetic field obtained in the last step, losses and reflections can be calculated. With the conductivity $\sigma$, the losses are computed by superposing (15) and (17) together. As for the reflections, we could get the S-matrix of the SWS by using (20) - (23). With the losses and reflections, the electric and magnetic field is provided in the SWS at time step $k$ are obtained, which are marked as $\boldsymbol{E}_{\boldsymbol{k}}$ and $\boldsymbol{H}_{\boldsymbol{k}}$, respectively.

Finally, the movement of particles and the power exchanged between beam and wave are calculated with the data of the computed electromagnetic field. The new velocity at time step $k+1 / 2$ marked as $\boldsymbol{v}_{\mathbf{k}+1 / 2}$ and the displacement in time step $k$ marked as $\boldsymbol{s}_{\boldsymbol{k}}$ are obtained from the previous velocity at time step $k-1 / 2$ marked as $\boldsymbol{v}_{\mathrm{k}-1 / 2}$ and the electric and magnetic field at time step $k, \boldsymbol{E}_{\boldsymbol{k}}$ and $\boldsymbol{H}_{\boldsymbol{k}}$, by Boris method. With the displacement $\boldsymbol{s}_{\boldsymbol{k}}$ and the electric field $\boldsymbol{E}_{\boldsymbol{k}}$, we obtain the exchanged power between beam and wave at time step $k$, marked as $\Delta P_{k}$, by using (27).

The new power value will be used to update the field. This process is repeat for the circuit until the end of the preset simulation time $t$.

\section{SIMULATION RESULTS AND DISCUSSIONS}

To verify the proposed theory and algorithm, a single section G-band $0.205 \mathrm{THz}$ staggered double vane TWT with 40 periods [2-4] is simulated by both this method and CST particle studio. The convergence of this algorithm is presented. The simulation times for the different methods are discussed.

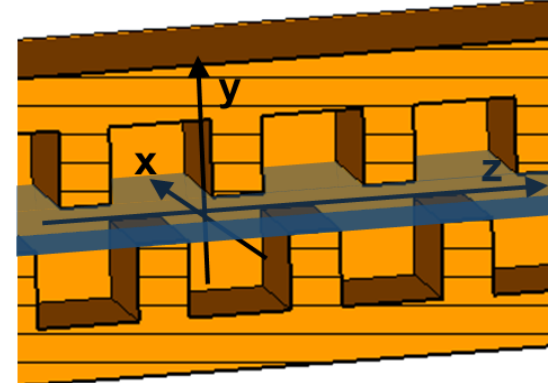

Fig.4. Schematic of G-band staggered double vane TWT with coordinate system [2].

\section{A. Model for Validations}

A schematic of a section of the staggered double vane SWS with coordinate system is shown in Fig.4. The width and height of beam channel are $0.8 \mathrm{~mm}$ and $0.15 \mathrm{~mm}$, respectively. The length of one period is $0.54 \mathrm{~mm}$. The depth and width of grating are $0.28 \mathrm{~mm}$ and $0.18 \mathrm{~mm}$, respectively. Oxygen-free copper is used as the conductor of this TWT, assuming a reduced conductivity $\sigma=2 \times 10^{7} \mathrm{~S} / \mathrm{m}$. The G-band TWT operates at $25 \mathrm{kV}$ beam voltage with $80 \mathrm{~mA}$ beam current. The input power is $50 \mathrm{~mW}$. The coupling impedance values of the first quadrant of the beam tunnel section of the G-band SWS in Fig. 4 computed by HFSS are shown in table I. The dimensional parameters of validation model are shown in table II.

\section{B. Simulation Results}

The macroparticles are emitted as a $13 \times 6$ array. The reflections caused by the discontinuities in the SWS are considered in the discussed example. The metal losses are not considered in simulation of S-matrix to avoid double counting, being included in calculation of losses in Sec. II.

TABLE I

COUPLing IMPEDANCE VAlue Of THE G-BAND STAGgered Double VANE SWS COMPUTED FOR THE FIRST QUADRANT (UNIT: $\Omega$ )

\begin{tabular}{|c|c|c|c|c|c|}
\hline $\mathrm{y} / \mathrm{mm}$ & 0 & 0.015 & 0.03 & 0.045 & 0.06 \\
\hline 0 & 4.3002 & 4.5277 & 4.9828 & 5.6653 & 6.5754 \\
\hline 0.04 & 4.0910 & 4.3075 & 4.7404 & 5.3898 & 6.2556 \\
\hline 0.08 & 3.6932 & 3.8886 & 4.2794 & 4.8656 & 5.6472 \\
\hline 0.12 & 3.1456 & 3.3120 & 3.6449 & 4.1442 & 4.8099 \\
\hline 0.16 & 2.5018 & 2.6342 & 2.8990 & 3.2961 & 3.8256 \\
\hline 0.2 & 1.8250 & 1.9215 & 2.1147 & 2.4043 & 2.7906 \\
\hline 0.24 & 1.1812 & 1.2437 & 1.3687 & 1.5562 & 1.8062 \\
\hline 0.28 & 0.6337 & 0.6672 & 0.7342 & 0.8348 & 0.9689 \\
\hline 0.32 & 0.2358 & 0.2483 & 0.2732 & 0.3107 & 0.3606 \\
\hline 0.36 & 0.0266 & 0.0280 & 0.0309 & 0.0351 & 0.0407 \\
\hline
\end{tabular}

TABLE II

Dimensional PARAMETERs Of A G-BAND STAGgered Double VANE SWS (UNIT: MM)

\begin{tabular}{cc}
\hline \hline Parameter & Value \\
\hline Width of Beam Channel & 0.8 \\
Length of Period & 0.54 \\
Depth of Vane & 0.28 \\
Length of Vane & 0.18 \\
Height of Beam Channel & 0.15 \\
\hline \hline
\end{tabular}

The comparison of S-parameters computed by the theory presented in Sec. III and by CST microwave studio is shown in Fig.5. Due to the structure symmetry, the curves of $S_{11}$ and $\mathrm{S}_{21}$ are presented. The $\mathrm{S}_{21}$ curves computed by the two methods are almost coincident above $0.21 \mathrm{THz}$ while the difference is less than $0.2 \mathrm{~dB}$ in the range of $0.19-0.21 \mathrm{THz}$. A good agreement is shown for the $\mathrm{S}_{11}$ curves. A $2-\mathrm{GHz}$ frequency offset is presented with difference between the two peak values around $6 \mathrm{~dB}$. In general, the S-parameters simulations show good agreement over the full frequency range.

Fig. 6 shows the power flow along the z-axis at $0.205 \mathrm{THz}$ computed by this method. The output power is $0.702 \mathrm{~W}(28.46$ $\mathrm{dBm})$, the gain is $11.47 \mathrm{~dB}$. 
Fig.7 shows the phase space diagram. The abscissa is the z-axial length of SWS, and the ordinate is the energy of each macroparticle. As we can see, consistent with the beam operation voltage, the energy of all macroparticles is $25 \mathrm{keV}$ at the beginning, while a good portion of macroparticles loss energy at the end of SWS, which results in the amplification of the electromagnetic wave.

Fig.8 shows the shape of the electron beam. The color represents the energy of macroparticles. To note that the beam after travelling uniform, after $10 \mathrm{~mm}$, diverges due to the space charge force.

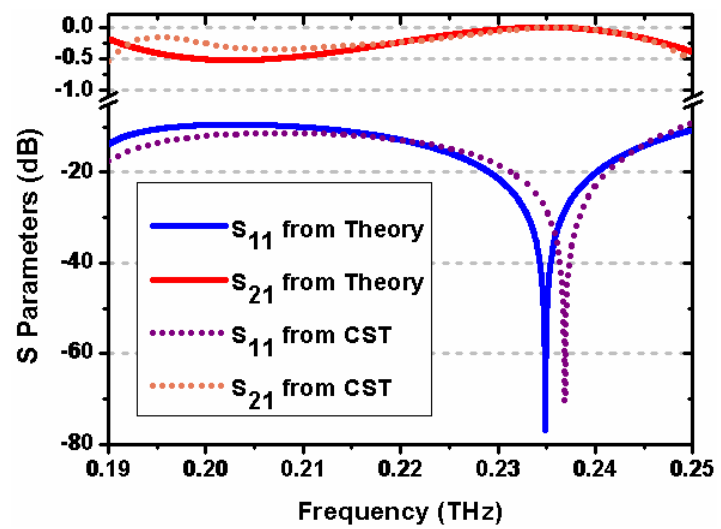

Fig. 5. The comparison of S-parameters between theory and CST.

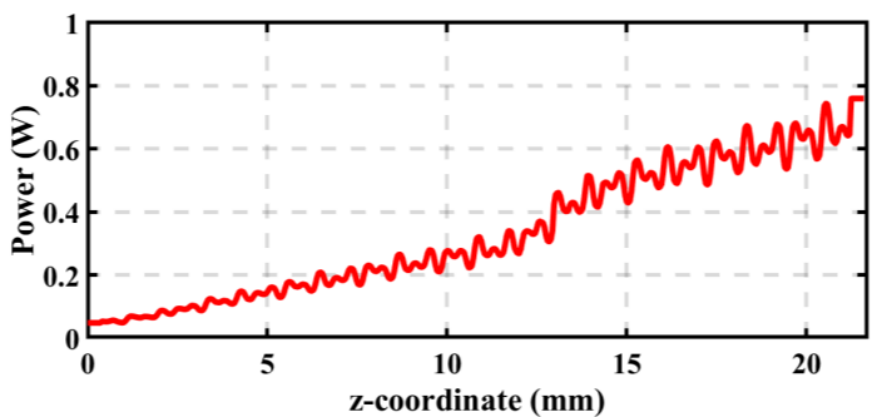

Fig. 6. The power flow along the z-axis.

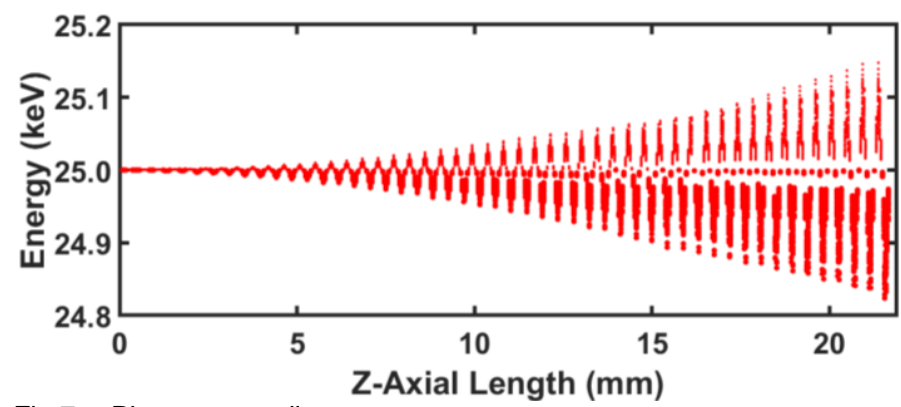

Fig.7. Phase space diagram.

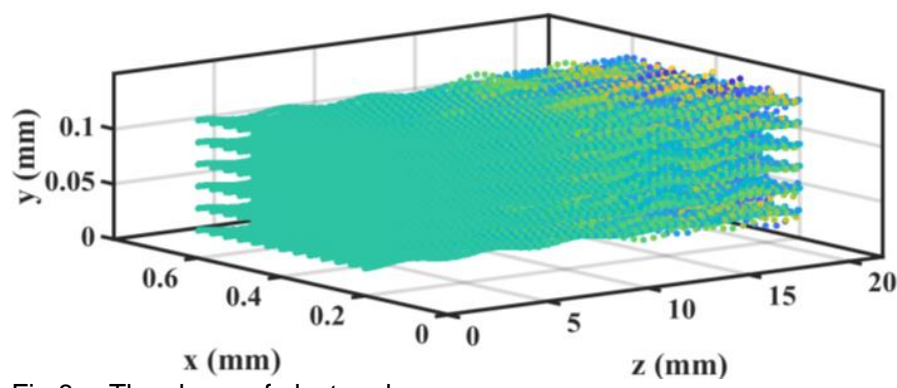

Fig.8. The shape of electron beam.

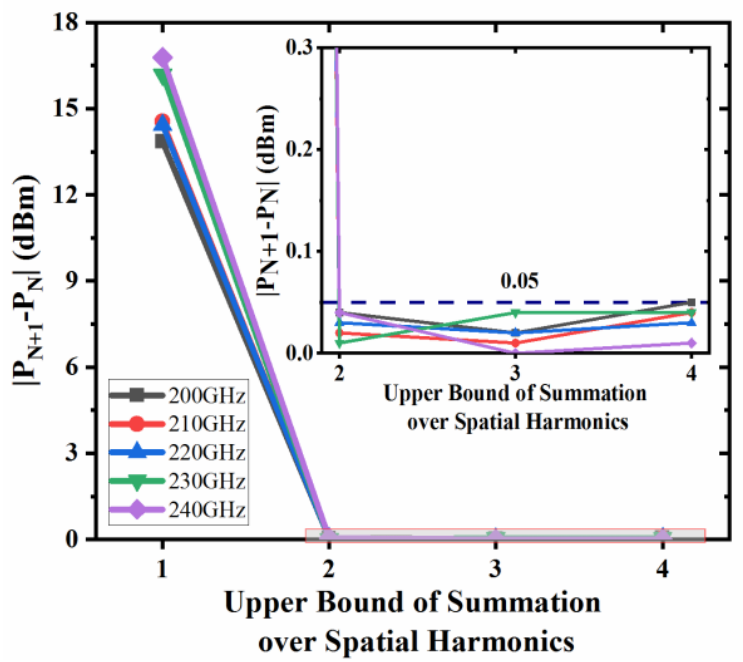

Fig. 9. The convergence characteristic of the algorithm.

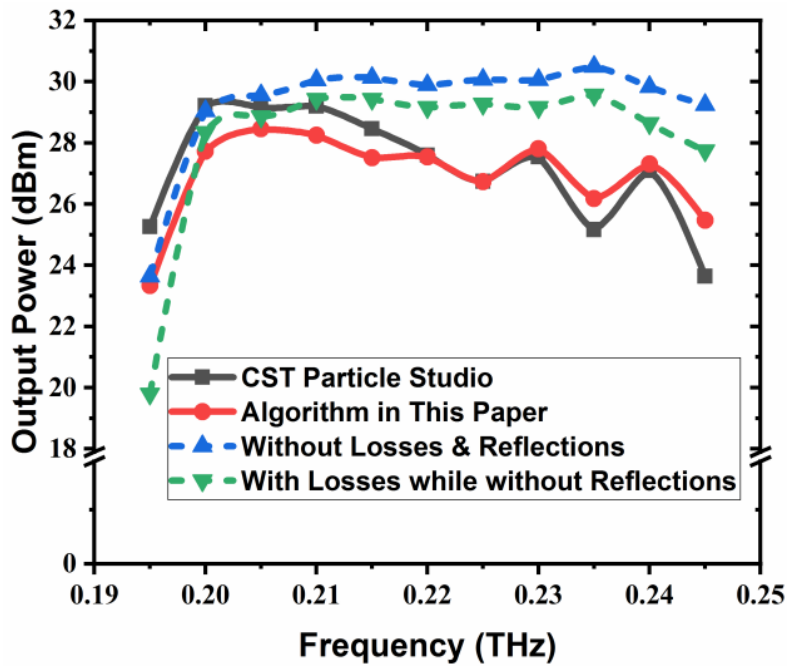

Fig. 10. Output power versus frequency, compared with the CST particle studio, the simulation without losses and reflections and the situation with losses but not reflections.

\section{The Convergence}

As shown in Section II, the electric and magnetic field are calculated by the superposition of spatial harmonics. Theoretically, this is an infinite series. In practice, however, only the first several spatial harmonics can be considered.

The more spatial harmonics are superimposed, the more accurate the results are. The calculation of too many spatial harmonics, however, increased the simulation time substantially. If the series of spatial harmonics is not convergent, no valid result will be obtained. Hence, it is important to study the convergence of the algorithm for its validation.

To study the convergence, the model described in Part A is used. The simulation results of output power versus frequency with $N$ from 1 to 5 are studied, where $N$ is the upper bound of summation over spatial harmonics in the superposition of electric and magnetic field described in Section II. The number of considered spatial harmonics are increasing with $\mathrm{N}$. Assuming $N=1$, only the $0^{\text {th }}$ spatial harmonic is considered. In case of $N=5,0^{\text {th }}, \pm 1^{\text {st }}$ and $\pm 2^{\text {nd }}$ spatial harmonics are considered.

The simulation results at sample frequency values in the 
band are shown in Fig.9. The axis of ordinates is the absolute value of the difference of two simulation results of output power with the upper bound $N$ and $N+1$ over spatial harmonics, and the axis of abscissa is the upper bound number over spatial harmonic. As we can see, the results converge rapidly when $N \geq 2$. The absolute value of the difference between two results is always no more than $0.05 \mathrm{dBm}$, which shows an excellent convergence.

Finally, the algorithm presented in this paper is convergent for the spatial harmonics, and reliable and stable for a given $N$. In this paper, we choose $N=4$.

\section{Comparisons and Discussions}

The same staggered double vane TWT has been simulated by the CST particle studio to be compared with this method over the $0.195-0.245 \mathrm{THz}$ band. For estimating the influence of the losses and reflections, this structure is also simulated in two other cases. The first case does not include losses and reflections, the second one, includes losses but not reflections. The output power versus frequency is shown in Fig.10. The comparison of the presented method with the CST particle studio shows very good agreement.

TABLE III

SETTING AND Run TIME FOR THE SAME EXAMPLE AT 0.195THz

\begin{tabular}{cccc}
\hline \hline & Algorithm in & & \\
& This Paper & FTDNA-3D & CST-PS \\
\hline Setting Time & $45 \mathrm{~min}$ & $45 \mathrm{~min}$ & $2 \mathrm{~h}$ \\
Run Time & $55.73 \mathrm{~min}$ & $39.59 \mathrm{~min}$ & $14.2 \mathrm{~h}$ \\
\hline \hline
\end{tabular}

To note the output power with losses and reflections at $0.195 \mathrm{THz}$ is less than in the case without them. Due to the TWT is oversaturated at this frequency, the bunches of electron beam will enter into deceleration phase before they leave the SWS. A stronger electromagnetic field will let the bunches of electron beam to enter into the deceleration phase earlier. In other words, the stronger electrical field is, the more oversaturated TWT. Without losses and reflections, the field is much stronger as expected. Thus, the blue curve is lower than red curve at $0.195 \mathrm{THz}$ in Fig. 10.

Table III shows the comparison of the approximate simulation time for the same structure at $0.195 \mathrm{THz}$ among the algorithm presented in this paper, the FTDNA-3D and CST particle studio, including setting time and run time. The computation time is for a computer with Intel Core i7-4790 CPU without GPU. The setting time for the algorithm in this paper and FTDNA-3D are including the time we spend to get the input parameters, such as Pierce impedance and phase constant. The setting time for the CST particle studio includes the time to implement the model and the simulation parameters.

\section{CONCLUSIONS}

An improved FTDNA-3D model including ohmic losses and reflections of an electromagnetic field propagating in sheet beam TWT is presented. The very good accuracy and the fast simulation time of the new model has been demonstrated by comparison with a $3 \mathrm{D}$ electromagnetic simulator. The validity of this theory has been verified by applying this method to a G-band staggered double vane SWS with a sheet beam. The results demonstrated good accuracies and convergence. The model is specifically useful for the simulation of SWSs for millimeter waves TWT where 3D PIC simulators need long simulation time. The Pierce impedances used in this paper is simulated by HFSS.

\section{APPENDIX}

The relations between the $\mathrm{x}$-component of circuit magnetic field and the y-component, the z-component circuit magnetic field and the y-component circuit electric field [5] are respectively:

$$
\begin{aligned}
& H_{c y}=\frac{1}{h^{2}} \frac{\partial^{2} H_{c x}}{\partial x \partial y} \\
& H_{c z}=\frac{1}{h^{2}} \frac{\partial^{2} H_{c x}}{\partial x \partial z} \\
& E_{c y}=\frac{j \omega \mu}{h^{2}} \frac{\partial H_{c x}}{\partial z}
\end{aligned}
$$

The active Helmholtz equations are described in (A4).

$$
\begin{gathered}
\nabla^{2} \vec{E}_{s}+k^{2} \vec{E}_{s}=\mu \frac{\partial \vec{J}}{\partial t}+\nabla \frac{\rho}{\varepsilon} \\
\nabla^{2} \vec{H}_{s}+k^{2} \vec{H}_{s}=-\nabla \times \vec{J}
\end{gathered}
$$

where $\boldsymbol{J}$ is the current density of bunched electron beam, $\rho$ is the charge density, $k$ is the wave number in the free space and

$$
k^{2}=\omega^{2} \mu_{0} \varepsilon_{0}
$$

The electromagnetic field in a periodic structure obeys the Floquet theorem. In case of losses, the z-components of the space charge electric and magnetic field are:

$$
\begin{aligned}
& E_{s z}(x, y, z)=\sum_{n} E_{s z m n}(x, y, z) e^{-\gamma_{e n} z} \\
& H_{s z}(x, y, z)=\sum_{n} H_{s z m n}(x, y, z) e^{-\gamma_{e n} z}
\end{aligned}
$$

where the complex electron propagation constant $\gamma_{e n}$ is related to period length $p$.

$$
\gamma_{e n}=\gamma_{e 0}+j \frac{2 n \pi}{p}
$$

where $\gamma_{e 0}$ is related with normalized velocity of electron

$$
\begin{gathered}
\gamma_{e 0}=\alpha+j \frac{v_{0}}{c} \\
\frac{\partial H_{s z n}}{\partial y}-\frac{\partial H_{s y n}}{\partial x}=j \omega \varepsilon E_{s x n}+J_{x n} \\
\frac{\partial E_{s x n}}{\partial z}-\frac{\partial E_{s z n}}{\partial x}=-j \omega \mu H_{s y n} \\
\frac{\partial H_{s x n}}{\partial z}-\frac{\partial H_{s z n}}{\partial x}=j \omega \varepsilon E_{s y n}+J_{y n} \\
\frac{\partial E_{s z n}}{\partial y}-\frac{\partial E_{s y n}}{\partial z}=-j \omega \mu H_{s x n}
\end{gathered}
$$

Where, footnote $s$ is on behalf of space charge field, $n$ is on behalf of $n$th spatial harmonics, and footnotes $x, y, z$ is on behalf of $x$-, $y$-, $z$-components, respectively.

Some values of parameters in Fig. $2 b$ are calculated as follow. 


$$
\begin{gathered}
\frac{b}{b^{\prime}}=\frac{Y_{0}^{\prime}}{Y_{0}}=\frac{1}{2 \alpha} \\
\frac{B_{a}}{Y_{0}}=\frac{2 b^{\prime}}{\lambda_{g}}\left(\tan ^{-1} \frac{1}{\alpha}+\frac{\ln \sqrt{1+\alpha^{2}}}{\alpha}\right) \\
\frac{B_{b}}{Y_{0}} \approx 1.1 \frac{b^{\prime}}{\lambda_{g}}\left[1-0.227 \frac{b^{\prime}}{b}+0.008\left(\frac{b^{\prime}}{b}\right)^{2}\right] \quad \text { ( A10a ) } \\
\frac{B_{c}}{Y_{0}}=\frac{\lambda_{g}}{2 \pi b^{\prime}} \\
\frac{B_{d}}{Y_{0}} \approx \frac{b}{\lambda_{g}}\left[2 \ln \frac{e b}{2 b^{\prime}}+1.1 \frac{b^{\prime}}{b}-0.167\left(\frac{b^{\prime}}{b}\right)^{2}+0.008\left(\frac{b^{\prime}}{b}\right)^{3}\right] \quad \text { ( A10e ) }
\end{gathered}
$$

where, the parameters $b$ and $b^{\prime}$ can be found in Fig.2a.

\section{ACKNOWLEDGMENTS}

This work was supported in part by the China Scholarship Council, in part by the National Natural Science Foundation of China under Grant No. 61921002 and No. 61988102.

\section{REFERENCES}

[1] H. Tian, Z. Lu, W. Shao, Z. Wang, J. Ma, T He, H. Gong, Z. Duan, T. Tang, Y. Wei, J. Feng, Y. Gong, "3-D fast nonlinear simulation for beam-w.ave interaction of sheet beam traveling-wave tube," IEEE Trans. Electron Devices, vol. 66, no. 3, pp. 1504-1511, Mar. 2019, doi: 10.1109/TED.2018.2889652.

[2] X. Shi, Z. Wang, X. Tang, T. Tang, H. Gong, Q. Zhou, W. Bo, Y. Zhang, Z. Duan, Y. Wei, Y. Gong, J. Fneg, "Study on wideband sheet beam traveling wave tube based on staggered double vane slow wave structure," IEEE Trans. Plasma Sci., vol. 42, no. 12, pp. 3996-4003, DEC. 2014, doi: 10.1109/TPS.2014.2365582.

[3] Y. Shin, L. R. Barnett, N. C. Luhmann, "Experimental, Numerical and Analytical Studies of the Staggered Double Vane Structure for $\mathrm{THz}$ Application," in Proc. IEEE Int. Vac. Electron. Conf. (IVEC), Rome, Italy, APR. 2009, doi: 10.1109/IVELEC.2009.5193375.

[4] C. Ruan, M. Zhan, "Design of a G-band Sheet Electron Beam Travelling Wave Tube," in Proc. IEEE Int. Vac. Electron. Conf. (IVEC), Monterey, CA, USA, APR. 2016, doi: 10.1109/IVEC.2016.7561875.

[5] A. A. Maragos, Z. C. Ioannidis, and I. G. Tigelis, "Dispersion characteristics of a rectangular waveguide grating," IEEE Trans. Plasma Sci., vol. 31, no. 5, pp. 1075-1082, OCT. 2003, doi: 10.1109/TPS.2003.819015

[6] A. G. Rozhnev, N. M. Ryskin, T. A. Karetnikova, G. V. Torgashev, N. I. Sinitsyn, P. D. Shalayev, and A. A. Burtsev, "Studying characteristics of the slow-wave system of the traveling-wave tube with a sheet electron beam," Radiophys. Quantum Electron., vol. 56, no. 8-9, pp. 542-553, Jan. 2014. doi: 10.1007/s11141-014-9457-1

[7] W. Wang, "Microwave engineering technology," Beijing, China: National defense industry press, 2009, pp. 274-279.

[8] N. Marcuvitz, "Waveguide handbook", Reprint, Originally published: New York : McGraw-Hill, 1951, pp. 307-310.

[9] H. Fehske, R. Schneider, and A. Weiße, "The particle-in-cell method", in Computational many-particle physics, Berlin, Germen: Springer, 2008, pp. 163-164.

[10] S. Zenitani, and T. Umeda, "On the Boris solver in particle-in-cell simulation," Phys. Plasmas, vol. 25, no. 11, p. 112110, NOV. 2018, doi: 10.1063/1.5051077.

[11] J.-L. Vay, "Simulation of beams or plasmas crossing at relativistic velocity," Phys. Plasmas, vol. 15, no. 5, p. 056701, FEB. 2008, doi: 10.1063/1.2837054.

[12] J. Rafelski, "The Lorentz force", in Relativity matters, Cham, Switzerland: Springer, 2017, pp. 317-342. 